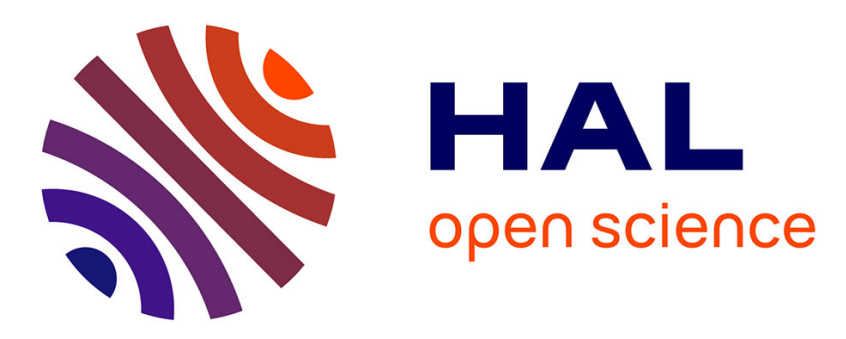

\title{
Maximal characterization of Hardy-Sobolev spaces on manifolds
}

\author{
Nadine Badr, Galia Dafni
}

\section{To cite this version:}

Nadine Badr, Galia Dafni. Maximal characterization of Hardy-Sobolev spaces on manifolds. International workshop on concentration, functional inequalities and isoperimetry, Oct 2009, United States. pp.10. hal-00863455

\section{HAL Id: hal-00863455 https://hal.science/hal-00863455}

Submitted on 19 Sep 2013

HAL is a multi-disciplinary open access archive for the deposit and dissemination of scientific research documents, whether they are published or not. The documents may come from teaching and research institutions in France or abroad, or from public or private research centers.
L'archive ouverte pluridisciplinaire HAL, est destinée au dépôt et à la diffusion de documents scientifiques de niveau recherche, publiés ou non, émanant des établissements d'enseignement et de recherche français ou étrangers, des laboratoires publics ou privés. 


\title{
Maximal characterization of Hardy-Sobolev spaces on manifolds
}

\author{
N. Badr and G. Dafni
}

\begin{abstract}
Let $M$ be a complete non-compact Riemannian manifold with a doubling measure and admitting a Poincaré inequality. In the present paper, we identify the Sobolev space $\dot{M}_{1}^{1}$, introduced by Hajłasz, with a new HardySobolev space defined by requiring the integrability of a certain maximal function of the gradient. This completes the circle of ideas begun in [4], where $\dot{M}_{1}^{1}$ was identified with a Hardy-Sobolev space via atomic decomposition.
\end{abstract}

\section{Introduction}

Let $M$ be a complete, non-compact Riemannian manifold, equipped with a doubling measure $\mu$. In previous work [4], we compared Hardy-Sobolev spaces on $M$, defined in terms of atomic decomposition, with an $L^{1}$-Sobolev space defined by Hajłasz $([\mathbf{1 1}]), M_{1}^{1}$, and showed they can be identified under the assumption of a Poincare inequality. The proof was based on a characterization of $M_{1}^{1}$ on metricmeasure spaces with doubling measures, by means of a Calderón-type Sobolev sharp maximal function $N f$, found in [15]. However, the most "natural" characterization remained to be shown: namely that, as in the Euclidean case, a function is in the Hardy-Sobolev space if its derivatives lie in the real Hardy space $H^{1}$, in the sense that a maximal function of the derivatives is integrable.

The problem arose in determining how to define the maximal function of the derivatives of $f$. In the Euclidean case, or the local case (i.e. on a chart on a compact manifold), one can consider the partial derivatives $\frac{\partial f}{\partial x_{j}}$, in the sense of distributions, and use the usual maximal function characterization of $H^{1}([\mathbf{1 9}],[\mathbf{1 6}])$. However, in the global case, i.e. on a non-compact Riemannian manifold, one would like to view the derivatives of $f$ in terms of the gradient $\nabla f$ or the differential $d f$. In [4] we identified $d f$ for $f \in M_{1}^{1}$ with elements of the molecular Hardy space of differential forms defined in [1], but maximal characterization there only holds under stronger geometric conditions.

2000 Mathematics Subject Classification. Primary 42B30, 46E35.

Project funded in part by the Natural Sciences and Engineering Research Council, Canada, and the the Centre de recherches mathématiques, Montréal.

(c) 0000 (copyright holder) 
Given a locally integrable function $f$ on $M$, one can define its gradient in the sense of distributions, by setting

$$
\langle\nabla f, \Phi\rangle:=-\int_{M} f \operatorname{div} \Phi d \mu
$$

for all smooth vector fields $\Phi$ of compact support. Following the ideas from the scalar case (see [21]), a natural ("grand") maximal function would be to take, at a point $x \in M$,

$$
\sup \left|\int_{M} f \operatorname{div} \Phi d \mu\right|
$$

where the supremum is taken over some family $\mathcal{T}_{1}(x)$ of test vector fields $\Phi$.

In order to do this, we need to extend the notion of divergence to a broader class of (minimally smooth) test vector fields. In [4], we defined a maximal function $(\nabla f)^{+}$(see Definition 3.1 below) where the test vector fields were, in a sense, only Lipschitz continuous. Moreover, it was shown (see [4], Proposition 5.3) that for $f \in L_{\text {loc }}^{1}(M),(\nabla f)^{+} \leq N f$ at every point of $M$, and therefore a function $f$ in the homogeneous Hajłasz Sobolev space $\dot{M}_{1}^{1}$, characterized by the condition $N f \in L^{1}$, also satisfies $(\nabla f)^{+} \in L^{1}$.

The difficulty arose in obtaining the converse, namely, showing that a function $f$ with $(\nabla f)^{+} \in L^{1}(M)$ belongs to $\dot{M}_{1}^{1}$, either by controlling $N f$ or via an atomic decomposition. In particular, when attempting to do this, the sticking point is the problem of writing a given test function $\eta$, with $\int \eta=0$, as the divergence of a sufficiently smooth vector field of compact support. In the Euclidean setting, this can be done by a simple well-known construction involving iterated integration with respect to the coordinates (see for example Lemma 6 in [16], or Lemma 4 in [5]) which preserves the smoothness with no gain. However, adapting such a construction to a manifold with constants which are independent of the local coordinates is not evident. In addition, if one wants to have a gain of derivatives, the case of $p=\infty$, which corresponds to starting with $\eta \in L^{\infty}$ and obtaining a vector field whose components have bounded derivatives, is not possible $([\mathbf{1 8}])$. In [5], the authors discuss existence of $L^{\infty}$ solutions in the endpoint case $\eta \in L^{d}$, where $d$ is the dimension.

Recently, in [8], this problem was studied again on arbitrary domains in $\mathbb{R}^{n}$, in weighted Sobolev spaces. We were able (see Proposition 5.1) to adapt the techniques used there to the case of a Riemannian manifold admitting a Poincaré inequality $\left(P_{1}\right)$, to obtain a solution of $\operatorname{div} \Phi=\eta$ with both $\eta$ and $\Phi$ in $L^{\infty}$. However, this does not give control on the derivatives of the components of $\Phi$ aside from its divergence, and therefore we can no longer restrict ourselves to "Lipschitz" vector fields as in Definition 3.1.

In Section 3, we give the definition of a new Hardy-Sobolev maximal function $\mathcal{M}^{+}(\nabla f)$, which generalizes that of the maximal function $M^{(1)} f$ used in [2] to characterize Hardy-Sobolev spaces on Lipschitz domains in $\mathbb{R}^{n}$, and use it to define the homogeneous maximal Hardy-Sobolev space $\dot{H} S_{\max }^{1}$. In Section 4, we compare this space with the homogeneous Hajłasz Sobolev space $\dot{M}_{1}^{1}$. Our main result, Theorem 4.4, is that under the assumption of a doubling condition and a Poincare inequality $\left(P_{1}\right)$, the two spaces coincide. The proof of the theorem, based on Proposition 5.1, is contained in Section 5. 


\section{Preliminaries}

We work on a complete non-compact Riemannian manifold $M$. With the distance function $\rho$ and the measure $\mu$ (volume) given by the Riemannian structure, we view $(M, \rho, \mu)$ as a metric measure space, and use $B(x, r)$ to denote the metric ball of radius $r>0$ centered at $x \in M$.

Denote by $\langle\cdot, \cdot\rangle_{x}$ the Riemannian metric on the the tangent space $T_{x} M$, let $T_{x}^{*} M$ be the cotangent space at $x$, and $d$ the exterior derivative. For a smooth function $f$, the gradient $\nabla f$ can be viewed as the image of the 1-form $d f$ under the isomorphism between $T_{x}^{*} M$ and $T_{x} M$ (see [22], Section 4.10). Given a smooth vector field $\boldsymbol{\Phi}$ with compact support, one can also define the divergence $\operatorname{div} \boldsymbol{\Phi} \in C^{\infty}(M)$ so that

$$
\int_{M}\langle\nabla f, \mathbf{\Phi}\rangle_{x} d \mu=-\int_{M} f \operatorname{div} \mathbf{\Phi} d \mu
$$

(see [20], Section 3.1.1).

We now extend this to a locally integrable function $f$ on $M$, in order to define $\nabla f$ in the sense of distributions, as in (1.1). If this distributional gradient coincides with a measurable vector-field, which we again denote by $\nabla f$, we can take its length in the Riemannian metric, $|\nabla f|_{x}:=\langle\nabla f, \nabla f\rangle_{x}^{1 / 2}$, and (dropping the subscript $x$ for simplicity) compute the semi-norms

$$
\|\nabla f\|_{p}:=\left(\int_{M}|\nabla f|^{p} d \mu\right)^{1 / p}, \quad 1 \leq p<\infty .
$$

The nonhomogeneous Sobolev space $W^{1, p}(M)$ is then defined as the space of $f$ in $L^{p}(M, \mu)$ with $\|\nabla f\|_{p}<\infty$. For the homogeneous Sobolev space $\dot{W}^{1, p}(M)$, we require only $f \in L_{\text {loc }}^{1}(M)$ with $\|f\|_{\dot{W}^{1, p}}:=\|\nabla f\|_{p}<\infty$, and consider the resulting space modulo constants.

A function will be called Lipschitz (continuous), denoted $f \in \operatorname{Lip}(M)$, if there exists $C<\infty$ such that

$$
|f(x)-f(y)| \leq C \rho(x, y) \quad \forall x, y \in M,
$$

and the smallest such constant $C$ will be denoted by $\|f\|_{\text {Lip }}$. By $\operatorname{Lip}_{0}(M)$ we will mean the space of compactly supported Lipschitz functions.

We will assume the measure $\mu$ on $M$ satisfies the following:

Definition 2.1 (Doubling Condition). There exists a constant $C>0$ such that for all balls $B(x, r), x \in M, r>0$, we have

$$
\mu(B(x, 2 r)) \leq C \mu(B(x, r)) .
$$

For certain results we will also assume:

Definition 2.2 (Poincaré inequality on $M$ ). We say that a Riemannian manifold $M$ admits a Poincaré inequality $\left(P_{q}\right)$ for some $q \in[1, \infty)$ if there exists a constant $C>0$ such that, for every every ball $B$ in $M$ of radius $r>0$,

$\left(P_{q}\right) \quad\left(f_{B}\left|f-f_{B}\right|^{q} d \mu\right)^{1 / q} \leq C r\left(f_{B}|\nabla f|^{q} d \mu\right)^{1 / q}$

whenever $f$ and its distributional gradient $\nabla f$ are $q$-integrable on $B$. 


\section{The maximal Hardy-Sobolev space}

Let us first recall the following definition:

Definition 3.1. ([4]) Let $f \in L_{\mathrm{loc}}^{1}(M)$. Define the maximal function $(\nabla f)^{+}$ by

$$
(\nabla f)^{+}(x):=\sup \left|\int f\left(\langle\nabla \varphi, \mathbf{\Phi}\rangle_{x}+\varphi \operatorname{div} \mathbf{\Phi}\right) d \mu\right|,
$$

where the supremum is taken over all pairs $\varphi \in \operatorname{Lip}_{0}(M), \mathbf{\Phi} \in C_{0}^{1}(M, T M)$ such that for some ball $B$ of radius $r$ containing $x$, we have

$$
\begin{gathered}
\operatorname{supp} \varphi \subset B, \quad\|\varphi\|_{\infty} \leq \frac{1}{\mu(B)}, \quad\|\nabla \varphi\|_{\infty} \leq \frac{1}{r \mu(B)}, \\
\|\boldsymbol{\Phi}\|_{\infty} \leq 1, \quad \text { and } \quad\|\operatorname{div} \boldsymbol{\Phi}\|_{\infty} \leq \frac{1}{r} .
\end{gathered}
$$

Note that in case both $\varphi$ and $\boldsymbol{\Phi}$ are smooth, the quantity $\langle\nabla \varphi, \boldsymbol{\Phi}\rangle_{x}+\varphi$ div $\boldsymbol{\Phi}$ represents the divergence of the product $\varphi \boldsymbol{\Phi}$, so the supremum on the right-handside is of $|\langle\nabla f, \varphi \Phi\rangle|$, as defined by (1.1). In fact, when we control the maximal function $(\nabla f)^{+}(x)$, we actually control the gradient of $f$, not just in the distributional sense but in the pointwise sense. This was shown by the following:

Proposition 3.2. (see Proposition 5.2 in [4]) Let $f \in L_{\mathrm{loc}}^{1}(M)$. If $(\nabla f)^{+} \in$ $L^{1}(M)$ then $\nabla f$, initially defined by (1.1), is given by an element of $L^{1}(M, T M)$ and satisfies

Consequently, $f \in \dot{W}^{1,1}(M)$ with

$$
|\nabla f|_{x} \leq C(\nabla f)^{+}(x) \quad \text { for } \mu \text { - a.e. } x \text {. }
$$

$$
\|f\|_{\dot{W}^{1,1}} \leq C\left\|(\nabla f)^{+}\right\|_{L^{1}}
$$

As explained in the introduction, the maximal function $(\nabla f)^{+}$appears to be too small since the test vector fields are assumed to have some Lipschitz smoothness. We want to relax the conditions on the vector fields even further.

Before defining the appropriate maximal function, it is necessary to extend the definition of divergence to non-smooth vector fields, namely to define it in a distributional sense. Following [8], which dealt with the case of a bounded domain in $\mathbb{R}^{n}$, we give the following definition.

Definition 3.3. Let $\Omega$ be a domain in $M, \mu(\Omega)<\infty$, and $\boldsymbol{\Phi}$ be a vector field in $L^{\infty}(\Omega, T M)$. We say that $\operatorname{div} \boldsymbol{\Phi}=g$ in the (strong) distributional sense if there exists $g \in L^{\infty}(\Omega)$ such that

$$
\int_{\Omega} f g d \mu=-\int_{\Omega}\langle\nabla f, \mathbf{\Phi}\rangle_{x} d \mu
$$

for all $f \in L_{\text {loc }}^{1}(M)$ with $f$ and its distributional gradient $\nabla f$ integrable on $\Omega$.

REMARK 3.4. (i) Taking $f$ to be a constant (since $\mu(\Omega)<\infty$ ), we must have $\int g d \mu=0$.

(ii) If $\boldsymbol{\Phi}$ is smooth with compact support in $\Omega$, then identity (3.2) holds for all $f \in C^{\infty}(\bar{\Omega})$ with $g=\operatorname{div} \boldsymbol{\Phi}$ in the strong sense. In the Euclidean case, if $\Omega$ has smooth boundary $\partial \Omega$, and $\nu$ is the normal to the boundary, then it suffices to require that $\left.\boldsymbol{\Phi} \cdot \nu\right|_{\partial \Omega}=0$. Requiring (3.2) to hold for $f$ without compact support is thus interpreted as $\boldsymbol{\Phi}$ satisfying a boundary condition (see Section 2 in [8] or 
[7] for a domain in the Euclidean case), hence the use of the word "strong" in the definition.

(iii) If $\varphi, \boldsymbol{\Phi}$ are as in Definition 3.1, with $B$ containing the support of $\varphi$, then for $f$ and $\nabla f$ integrable on $B$, we have that

$$
\nabla(f \varphi)=\varphi \nabla f+f \nabla \varphi
$$

Hence

$$
\int\langle\nabla f, \varphi \mathbf{\Phi}\rangle_{x} d \mu=-\int f\left(\langle\nabla \varphi, \boldsymbol{\Phi}\rangle_{x}+\varphi \operatorname{div} \mathbf{\Phi}\right) d \mu
$$

and $\operatorname{div}(\varphi \mathbf{\Phi})=\langle\nabla \varphi, \boldsymbol{\Phi}\rangle_{x}+\varphi \operatorname{div} \boldsymbol{\Phi}$ on $B$, in the sense of Definition 3.3.

Now we will define the new maximal homogeneous Hardy-Sobolev space $\dot{H} S_{\max }^{1}$.

Definition 3.5. We define the maximal homogeneous Hardy-Sobolev space $\dot{H} S_{\max }^{1}$ as follows:

$$
\dot{H} S_{\max }^{1}:=\left\{f \in L_{\mathrm{loc}}^{1}: \mathcal{M}^{+}(\nabla f) \in L^{1}\right\},
$$

where $\mathcal{M}^{+}(\nabla f)$ is given by

$$
\mathcal{M}^{+}(\nabla f)(x):=\sup \left|\int f \operatorname{div} \boldsymbol{\Phi} d \mu\right| .
$$

Here the supremum is taken over all $\boldsymbol{\Phi} \in \mathcal{T}_{1}(x)$, that is: $\boldsymbol{\Phi} \in L^{\infty}(B, T M)$ for some ball $B$ of radius $r$ containing $x$, such that there exists $g \in L^{\infty}(B), g=\operatorname{div} \boldsymbol{\Phi}$ in the sense of Definition 3.3, and

$$
\|\mathbf{\Phi}\|_{\infty} \leq \frac{1}{\mu(B)}, \quad\|g\|_{\infty} \leq \frac{1}{r \mu(B)} .
$$

We equip this space with the semi-norm

$$
\|f\|_{H S_{\max }^{1}}=\left\|\mathcal{M}^{+}(\nabla f)\right\|_{1} .
$$

Note that the definition of $\mathcal{M}^{+}(\nabla f)$ generalizes that of the maximal function $M^{(1)} f$ used in [2] to characterize Hardy-Sobolev spaces on Lipschitz domains in $\mathbb{R}^{n}$.

REMARK 3.6. By Remark 3.4(iii), we have that for $f \in L_{\text {loc }}^{1}(M),(\nabla f)^{+} \leq$ $\mathcal{M}^{+}(\nabla f)$, and thus Proposition 3.2 shows that for $f \in \dot{H} S_{\text {max }}^{1}$,

$$
|\nabla f| \leq C \mathcal{M}^{+}(\nabla f) \quad \mu-\text { a.e. }
$$

Consequently,

$$
\dot{H} S_{\max }^{1} \subset \dot{W}^{1,1}(M)
$$

with

$$
\|f\|_{\dot{W}^{1,1}} \leq C\|f\|_{\dot{H}^{1} \max ^{1}}
$$




\section{The Hajłasz Sobolev space}

The goal of this paper is to compare the maximal Hardy-Sobolev space with the Hajłasz Sobolev space, which can be defined on any metric-measure space $(X, d, \mu)$ :

Definition 4.1 (Hajłasz). Let $1 \leq p \leq \infty$. The (homogeneous) Sobolev space $\dot{M}_{p}^{1}$ is the space of all functions $u \in L_{\text {loc }}^{1}$ such that there exists a measurable function $g \geq 0, g \in L^{p}$, satisfying

$$
|u(x)-u(y)| \leq d(x, y)(g(x)+g(y)) \mu-a . e .
$$

We equip $\dot{M}_{p}^{1}$ with the semi-norm

$$
\|u\|_{\dot{M}_{p}^{1}}=\inf _{g \text { satisfies(4.1) }}\|g\|_{p} .
$$

Modulo constants, $\dot{M}_{p}^{1}$ becomes a Banach space. A nonhomogeneous version $M_{p}^{1}:=L^{p} \cap \dot{M}_{p}^{1}$ can be defined using the norm $\|u\|_{p}+\|u\|_{\dot{M}_{p}^{1}}$. For $p>1$, these spaces can be identified with the usual Sobolev spaces in the Euclidean case (see [11]) and are part of a more general theory of Sobolev spaces on metric-measure spaces (see [12],[14]). For $p=1$, in the Euclidean case, Koskela and Saksman [16] identified $\dot{M}_{1}^{1}\left(\mathbb{R}^{n}\right)$ with the homogeneous Hardy-Sobolev space $\dot{H} S^{1}\left(\mathbb{R}^{n}\right.$ ) (a more recent result [17] identifies $\dot{M}_{p}^{1}\left(\mathbb{R}^{n}\right), p>\frac{n}{n+1}$, with Triebel-Lizorkin spaces defined in terms of Littlewood-Paley decompositions).

Hardy-Sobolev spaces on $\mathbb{R}^{n}$ can be defined by requiring all partial derivatives of $f$ up to a certain order, taken in the sense of distributions, to lie in the real Hardy space $H^{p}$. These Hardy spaces can also be characterized, as was done by Gatto, Segovia and Jimenez [10], via a type of maximal function used by Calderón [6] in the case $p>1$, or by another maximal function, shown by Devore and Sharpley [9] to be pointwise equivalent to Calderón's maximal function, as in the work of Miyachi [19]. We now define this latter maximal function, which we call a Sobolev sharp maximal function, corresponding to the case of one derivative in $H^{1}$ :

Definition 4.2. For $f \in L_{\mathrm{loc}}^{1}$, define $N f$ by

$$
N f(x)=\sup _{B: x \in B} \frac{1}{r(B)} f_{B}\left|f-f_{B}\right| d \mu,
$$

where $B$ denotes a ball, $r(B)$ its radius and $f_{B}$ the average of $f$ over $B$.

Note that this definition also makes sense in any metric-measure space. In this more general setting, but assuming a doubling condition, Kinnunen and Tuominen [15] show that $\dot{M}_{1}^{1}$ consists of those functions for which $\mathrm{Nf}$ (called a "fractional sharp maximal function" in [15]), is integrable. This characterization extends to $p=1$ a previous result of Hajłasz and Kinnunen [13] for $p>1$ :

TheOREm $4.3([\mathbf{1 5}])$. On a metric space with metric d and doubling measure $\mu$,

with

$$
\dot{M}_{1}^{1}=\left\{f \in L_{\mathrm{loc}}^{1}: N f \in L^{1}\right\}
$$

$$
\|f\|_{\dot{M}_{1}^{1}} \sim\|N f\|_{1} .
$$

Moreover, if $f \in L_{\mathrm{loc}}^{1}$ and $N f \in L^{1}$, then $f$ satisfies

$$
|f(x)-f(y)| \leq C d(x, y)(N f(x)+N f(y))
$$

for $\mu$-a.e. $x, y$. 
Using this characterization, in our previous work [4] we were able to identify the Hajłasz Sobolev spaces $\dot{M}_{1}^{1}$ and $M_{1}^{1}$ with the (homogeneous and nonhomogeneous) atomic Hardy-Sobolev spaces studied in [3]. The following result completes this circle:

TheOREM 4.4. Let $M$ be a complete Riemannian manifold satisfying $(D)$. Suppose $f \in L_{\text {loc }}^{1}$. Then at every point of $M$,

$$
\mathcal{M}^{+}(\nabla f) \leq N f
$$

Consequently,

$$
\dot{M}_{1}^{1} \subset \dot{H} S_{\max }^{1}
$$

with

$$
\|f\|_{\dot{H}^{\prime} \max ^{1}} \leq C\|f\|_{\dot{M}_{1}^{1}}
$$

If moreover we assume $\left(P_{1}\right)$, then

$$
\mathcal{M}^{+}(\nabla f) \approx N f
$$

and

$$
\dot{H} S_{\max }^{1}=\dot{M}_{1}^{1}
$$

\section{Proof of Theorem 4.4}

Proof. Let $f \in L_{\mathrm{loc}}^{1}$ and $x \in M$. Take $\boldsymbol{\Phi} \in \mathcal{T}_{1}(x)$, as in Definition 3.5, associated to a ball $B$ containing $x$. Then as pointed out in Remark 3.4(i),

$$
\int \operatorname{div} \boldsymbol{\Phi} d \mu=0
$$

so we can write

$$
\begin{aligned}
\left|\int f \operatorname{div} \mathbf{\Phi} d \mu\right| & =\left|\int_{B}\left(f-f_{B}\right) \operatorname{div} \mathbf{\Phi} d \mu\right| \\
& \leq \frac{1}{r \mu(B)} \int_{B}\left|f-f_{B}\right| d \mu \\
& \leq N f(x) .
\end{aligned}
$$

Here $r$ is the radius of $B$. Taking the supremum over all such $\boldsymbol{\Phi}$, we get (4.3).

We proceed now to the proof of the reverse inequality. For this, we will need the following:

Proposition 5.1. Let $M$ be a complete Riemannian manifold satisfying $(D)$ and $\left(P_{1}\right)$. Let $B$ a ball of $M$,

$$
g \in L_{0}^{\infty}(B):=\left\{g \in L^{\infty}(B): \int_{B} g d \mu=0\right\} .
$$

Then there exists $\boldsymbol{\Phi} \in L^{\infty}(B, T M)$ such that

$$
\operatorname{div} \boldsymbol{\Phi}=g
$$

holds in the sense of Definition 3.3 (with $\Omega=B$ ), and

$$
\|\mathbf{\Phi}\|_{\infty} \leq C r\|g\|_{\infty}
$$

where $C$ is the constant appearing in $\left(P_{1}\right)$ and is independent of $B$ and $g$. 
Before proving the proposition, we conclude the proof of Theorem 4.4. Again take $f \in L_{\text {loc }}^{1}, x \in M$, and $B$ a ball of radius $r$ containing $x$. If $g \in L_{0}^{\infty}(B)$, $\|g\|_{\infty} \leq 1$, and we solve $\operatorname{div} \boldsymbol{\Phi}=g$ with $\boldsymbol{\Phi}$ as in Proposition 5.1, then (with the same constant $C$ ) the vector field

$$
\widetilde{\mathbf{\Phi}}:=\frac{\mathbf{\Phi}}{\operatorname{Cr\mu }(B)} \in \mathcal{T}_{1}(x),
$$

and

$$
\left|\int_{B} f g d \mu\right|=\left|\int_{B} f \operatorname{div} \mathbf{\Phi} d \mu\right|=C r \mu(B)\left|\int_{B} f \operatorname{div}(\widetilde{\mathbf{\Phi}}) d \mu\right|
$$

Thus

$$
\begin{aligned}
\frac{1}{r \mu(B)} \int_{B}\left|f-f_{B}\right| d \mu & =\frac{1}{r \mu(B)} \sup _{g \in L_{0}^{\infty}(B),\|g\|_{\infty} \leq 1}\left|\int_{B} f g d \mu\right| \\
& \leq C \sup _{\widetilde{\mathbf{\Phi}} \in \mathcal{T}_{1}(x)}\left|\int f \operatorname{div}(\widetilde{\mathbf{\Phi}}) d \mu\right| \\
& =C \mathcal{M}^{+}(\nabla f)(x) .
\end{aligned}
$$

Taking the supremum on the left over all balls $B$ containing $x$, we get $N f(x) \leq$ $C \mathcal{M}^{+}(\nabla f)(x)$.

Proof of Proposition 5.1. The proof follows that of Proposition 3.2 in [8] in the Euclidean weighted case. Let $B$ be a ball and $g \in L_{0}^{\infty}(B)$. Consider

$$
\mathcal{S}:=\left\{V \in L^{1}(B, T M): \exists f \in L_{\text {loc }}^{1}(M), V=\nabla f \text { on } B\right\} .
$$

We view $\mathcal{S}$ as a subspace of $L^{1}(B, T M)$ with the norm

$$
\|V\|_{L^{1}(B, T M)}=\int_{B}|V|_{x} d \mu
$$

Define a linear functional on $\mathcal{S}$ by

$$
\Lambda(V)=-\int_{B} g f d \mu \quad \text { if } V=\nabla f \in \mathcal{S} .
$$

This functional is well defined since $\int_{B} g d \mu=0$ (the Poincaré inequality implies that on $B$, if $V=\nabla f=0$ then $f$ must be constant), and $\Lambda$ is bounded on $\mathcal{S}$, again thanks to the Poincaré inequality $\left(P_{1}\right)$ :

$$
|\Lambda(V)|=\left|\int_{B} g\left(f-f_{B}\right) d \mu\right| \leq C r\|g\|_{\infty} \int_{B}|\nabla f| d \mu=C r\|g\|_{\infty}\|V\|_{L^{1}(B, T M)} .
$$

The Hahn-Banach theorem shows that $\Lambda$ can be extended to a bounded linear functional on $L^{1}(B, T M)$ with norm no greater than $C r\|g\|_{\infty}$. By duality, there exists a vector field $\boldsymbol{\Phi} \in L^{\infty}(B, T M)$ such that

$$
\int_{B}\langle\Phi, \nabla f\rangle_{x} d \mu=\Lambda(\nabla f)=-\int_{B} g f d \mu
$$

for all $f \in L_{\text {loc }}^{1}(M)$ for which $\nabla f \in L^{1}(B, T M)$. By Definition 3.3, this means $\operatorname{div} \boldsymbol{\Phi}=g$ on $B$. Moreover,

$$
\|\mathbf{\Phi}\|_{\infty} \leq C r\|g\|_{\infty}
$$




\section{References}

1. P. Auscher, A. McIntosh, E. Russ, Hardy spaces of differential forms on Riemannian manifolds, J. Geom. Anal. 18 (2008), 192-248.

2. P. Auscher, E. Russ, P. Tchamitchian, Hardy Sobolev spaces on strongly Lipschitz domains of $\mathbb{R}^{n}$, J. Funct. Anal. 218 (2005), 54-109.

3. N. Badr, F. Bernicot, Abstract Hardy-Sobolev spaces and interpolation, J. Funct. Anal. 259 (2010), 1169-1208.

4. N. Badr, G. Dafni, An atomic decomposition of the Hajłasz Sobolev space on manifolds, J. Funct. Anal. 259 (2010), 1380-1420.

5. J. Bourgain, H. Brezis, On the equation $\operatorname{div} Y=f$ and application to control of phases, J. Amer. Math. Soc. 16 (2003), 393-426.

6. A. P. Calderón, Estimates for singular integral operators in terms of maximal functions, in: Collection of articles honoring the completion by Antoni Zygmund of 50 years of scientific activity, VI, Studia Math. 44 (1972), 563-582.

7. D.-C. Chang, G. Dafni, C. Sadosky, A div-curl lemma in BMO on a domain, in "Harmonic analysis, signal processing, and complexity", 55-65, Progr. Math. 238, Birkhäuser Boston, Boston, MA, 2005.

8. R. Duran, M.-A. Muschietti, E. Russ, P. Tchamitchian, Divergence operator and Poincaré inequalities on arbitrary bounded domains, Complex Var. Elliptic Equ. 55 (2010) 795-816.

9. R. A. DeVore, R. C. Sharpley, Maximal functions measuring smoothness, Mem. Amer. Math. Soc. $47,1984$.

10. A. E. Gatto, C. Segovia, J. R. Jiménez, On the solution of the equation $\Delta^{m} F=f$ for $f \in H^{p}$, Conference on harmonic analysis in honor of Antoni Zygmund, Vol. I, II (Chicago, Ill., 1981), 394-415, Wadsworth Math. Ser., Wadsworth, Belmont, CA, 1983.

11. P. Hajłasz, Sobolev spaces on an arbitrary metric space, Potential Anal. 5 (1996), 403-415.

12. P. Hajłasz, Sobolev spaces on metric-measure spaces, in: Heat kernels and analysis on manifolds, graphs, and metric spaces (Paris, 2002), Contemp. Math. 338, Amer. Math. Soc. Providence, RI 2003, pp. 173-218.

13. P. Hajłasz, J. Kinnunen, Hölder quasicontinuity of Sobolev functions on metric spaces, Rev. Mat. Iberoam. 14 (1998), 601-622.

14. P. Hajłasz, P. Koskela, Sobolev met Poincaré, Mem. Amer. Math. Soc. 145 (2000), 1-101.

15. J. Kinnunen, H. Tuominen, Pointwise behaviour of $M^{1,1}$ Sobolev functions, Math. Z. 257 (2007), 613-630.

16. P. Koskela, E. Saksman, Pointwise characterizations of Hardy-Sobolev functions, Math. Res. Lett. 15 (2008), 727-744.

17. P. Koskela, D. Yang, Y. Zhou, A characterization of Hajłasz-Sobolev and Triebel-Lizorkin spaces via grand Littlewood-Paley functions, J. Funct. Anal. 258 (2010), 2637-2661.

18. C. T. McMullen, Lipschitz maps and nets in Euclidean space, Geom. Funct. Anal. 8 (1998), 304-314.

19. A. Miyachi, Hardy-Sobolev spaces and maximal functions, J. Math. Soc. Japan 42 (1990), 73-90.

20. L. Saloff-Coste, Aspects of Sobolev-Type Inequalities, London Mathematical Society Lecture Note Series 289, Cambridge University Press, Cambridge, UK, 2002.

21. E. M. Stein, Harmonic analysis: real-variable methods, orthogonality, and oscillatory integrals, Princeton Mathematical Series 43, Princeton University Press, Princeton, NJ, 1993.

22. F. W. Warner, Foundations of Differentiable Manifolds and Lie Groups, Scott, Foresman and Company, Glenview, Illinois, 1971.

Université de Lyon ; CNRS ; Université Lyon 1, Institut Camille Jordan, 43 boulevard du 11 novembre 1918, F-69622 Villeurbanne Cedex, France.

E-mail address: badr@math.univ-lyon1.fr

Department of Mathematics and Statistics, Concordia University, 1455 de Maisonneuve Blvd. West, Montréal, QC, Canada H3G1M8

E-mail address: gdafni@mathstat.concordia.ca 